\section{Desafios e avanços no controle de resíduos de agrotóxicos no Brasil: 15 anos do Programa de Análise de Resíduos de Agrotóxicos em Alimentos}

\author{
Challenges and strides in the control of pesticide \\ residues in Brazil: 15 years of the Program for \\ Analysis of Pesticide Residues in Food Products
}
Desafíos y avances en el control de residuos de pesticidas en Brasil: 15 años del Programa de Análisis de Residuos de Pesticidas en Alimentos

\section{Resumo}

No Brasil, desde 2001, o monitoramento da presença de agrotóxicos nos alimentos ocorre por meio do Programa de Análise de Resíduos de Agrotóxicos em Alimentos (PARA). O objetivo foi analisar os desafios e avanços no controle de resíduos de agrotóxicos nos alimentos, no Brasil, com base nos resultados do PARA, entre os anos 2001 e 2015. Foi realizada pesquisa descritivo-analítica documental, que utilizou dados provenientes dos relatórios do PARA, disponíveis no sítio eletrônico da Agência Nacional de Vigilância Sanitária. Entre os principais desafios apresentados pelo PARA estão a grande variabilidade no número de amostras e ingredientes ativos analisados, bem como no formato dos relatórios apresentados. Existe a necessidade do aprofundamento das discussões a respeito dos critérios usados para a classificação e autorização dos ingredientes ativos no Brasil, bem como da transparência destas informações para a sociedade. No total, foram analisados 25 tipos de alimentos e mais de 30 mil amostras ao longo da vigência do PARA, porém, o máximo de ingredientes ativos analisados foi 228. A presença de ingredientes ativos não autorizados para a cultura e não autorizados no país foi a principal causa de irregularidades diagnosticadas nos alimentos. Embora ainda existam diversos desafios apresentados no PARA, ele contribui para disparar a reflexão sobre o tema junto à sociedade. Haja vista a atual conjuntura do governo, de maior flexibilização na liberação de ingredientes ativos no país, é necessário que o PARA seja fortalecido.

Agrotóxicos; Segurança Alimentar e Nutricional; Vigilância Sanitária
Carla Vanessa Alves Lopes 1

Guilherme Souza Cavalcanti de Albuquerque 1

doi: 10.1590/0102-311X00116219

Correspondência

C. V. A. Lopes

Universidade Federal do Paraná.

Rua Padre Camargo 280, 7o andar NESC, Curitiba, PR 80060-240, Brasil.

carla.valopes.ufpr@gmail.com

1 Universidade Federal do Paraná, Curitiba, Brasil. 


\section{Introdução}

O impacto negativo à saúde humana e ao meio ambiente causado pela exposição aguda e crônica aos agrotóxicos já é bem conhecido pela literatura e, apesar disto, contraditoriamente, o Brasil consome imensas quantidades destes venenos, ficando entre os maiores consumidores de agrotóxicos do mundo ${ }^{1}$.

No meio ambiente, o efeito do uso intensivo de agrotóxicos causa desde a degradação do solo e da água 2 até a contaminação de organismos aquáticos 3 e terrestres 4 , podendo até mesmo levar à letalidade, fato frequente, na atualidade, em relação às abelhas 5,6. Na saúde humana, a exposição a esses venenos pode ocorrer por meio de absorção dérmica, inalação ou por intermédio de alimentos contaminados 7, causando desde intoxicações agudas 8 até efeitos tardios como alguns tipos de câncer 9,10. Um estudo conduzido no Paraná identificou, em um total de 46 indivíduos, 20 casos de intoxicação crônica de origem laboral por agrotóxicos em fumicultores, incluindo transtornos psiquiátricos menores, perdas auditivas e polineuropatia tardia, causados por organofosforados 11 .

Uma extensa revisão sistemática sobre o impacto dos agrotóxicos na saúde humana e ambiental demonstra que a exposição aos agrotóxicos pode também estar associada a danos em mecanismos de defesa celular, suicídio, dores no corpo, depressão, ansiedade, distúrbios respiratórios, linfoma não Hodgkin, óbitos fetais, alterações hepáticas e hormonais, alterações em sistemas reprodutores masculinos e femininos, e perdas auditivas 12 .

Estudos mostram que alimentos expostos para o consumo humano também podem estar contaminados por resíduos dessas substâncias utilizadas no campo 13,14. Além disso, a composição nutricional dos alimentos contaminados por agrotóxicos pode ser alterada, de forma a reduzir, por exemplo, sua própria quantidade de antioxidantes 15.

Longe da intenção de eliminar o uso dessas substâncias na produção de alimentos, programas e políticas públicas são implantados com a pretensão de monitorar o uso de agrotóxicos no Brasil e orientar o seu uso, o que reduziria o risco à saúde causado pelos venenos. $\mathrm{Na}$ área de saúde do trabalhador, por exemplo, as medidas preconizadas incluem o uso de equipamentos de proteção individual (EPI), ações educativas e boas práticas agrícolas 16 .

Na área de alimentos, a Agência Nacional de Vigilância Sanitária (Anvisa) implantou, desde 2001, o Programa de Análise de Resíduos de Agrotóxicos em Alimentos (PARA). O PARA coleta, periodicamente, com o apoio das equipes de vigilância sanitária municipais e estaduais, alimentos expostos à venda em redes de supermercados distribuídos nos municípios do território nacional, para que sejam analisados nos diversos laboratórios públicos e privados contratados. A análise consiste em identificar os tipos de agrotóxicos presentes nos alimentos, com base no método de multiresíduos e outros métodos específicos para algumas substâncias, e verificar se são autorizados para aquela cultura e se estão dentro do limite máximo de resíduos (LMR) permitido pela Anvisa 17.

A definição dos alimentos que são analisados pelo PARA baseia-se nos estudos de consumo alimentar da população brasileira. Já a escolha de grandes redes de supermercados como pontos de coleta é orientada, em primeiro lugar, pela disponibilidade de diversos tipos de alimentos nestes locais e pela recomendação de que os produtos sejam provenientes do último ponto da cadeia antes do consumo 17.

A análise das informações produzidas por programas governamentais, como o PARA, é de extrema relevância para a qualificação da gestão e o aprimoramento destes programas, especialmente para que os pontos frágeis sejam minorados.

O presente trabalho tem como objetivo analisar os desafios e avanços no controle de resíduos de agrotóxicos nos alimentos no Brasil, baseando-se nos resultados do PARA, entre os anos 2001 e 2015.

\section{Metodologia}

Trata-se de uma pesquisa descritivo-analítica documental, que utilizou dados provenientes dos relatórios do PARA, disponíveis no sítio eletrônico da Anvisa (https://www.gov.br/anvisa/pt-br). Foram selecionados todos os relatórios do PARA, desde a sua implantação, em 2001, até o último relatório, publicado em 2016. 
Foram extraídos dos relatórios os dados referentes aos tipos de alimentos analisados, o número de amostras de alimentos analisadas, o número de ingredientes ativos monitorados, o número de ingredientes ativos detectados, a porcentagem de ingredientes ativos não autorizados para a cultura e a porcentagem de ingredientes ativos, cujos níveis encontravam-se dentro e acima do LMR.

A Portaria no 03/1992 da Secretaria de Vigilância em Saúde/Ministério da Saúde 18 apresenta o conceito de LMR como: "quantidade máxima de resíduo de agrotóxico legalmente aceita no alimento, em decorrência da aplicação adequada numa fase específica, desde a sua produção até o consumo, expressa em parte (em peso) do agrotóxico ou seus derivados por um milhão de partes de alimento (em peso) (ppm ou mg/kg)". O cálculo do LMR é baseado na ingestão diária aceitável (IDA), ou seja, a soma do LMR de um ingrediente ativo encontrado nas diversas culturas em que é autorizado o seu uso não pode ultrapassar a IDA 19.

$\mathrm{Na}$ avaliação toxicológica para o estabelecimento da IDA são acurados os efeitos biológicos, bioquímicos e toxicológicos em animais de laboratório e, depois, inferidos os riscos para a saúde humana. A IDA, expressa em $\mathrm{mg}$ do agrotóxico por $\mathrm{kg}$ de peso corpóreo, é considerada a quantidade máxima que, ingerida diariamente durante toda a vida, parece não oferecer risco apreciável à saúde 18 .

Além da avaliação da toxicidade crônica, a dose de referência aguda (DRfA), também expressa em mg do resíduo por kg do peso corpóreo, representa a quantidade estipulada do agrotóxico presente no alimento que, em um período de 24 horas, não causa efeito adverso à saúde 17 .

Os diferentes tipos de alimentos analisados pelo PARA são determinados, a cada ano, com base nos dados de consumo de alimentos obtidos na Pesquisa de Orçamentos Familiares (POF), bem como na disponibilidade dos alimentos nos supermercados ${ }^{17}$. Já a quantidade de amostras analisadas depende diretamente da capacidade analítica dos laboratórios e do transporte das amostras íntegras até eles. Quanto à escolha dos ingredientes ativos pesquisados, o PARA baseia-se nas informações sobre a utilização de agrotóxicos no Brasil, nos dados disponibilizados pelos laboratórios quanto aos resíduos usualmente detectados nos alimentos, e na disponibilidade de método analítico pelos laboratórios 17,20.

Os dados encontrados nos relatórios foram coletados no período de julho a dezembro de 2016, tabulados em planilhas do Microsoft Excel (https://products.office.com/), separadas por tipo de alimento, em ordem cronológica, de forma a identificar cada agrotóxico detectado ao longo dos anos do PARA.

Após a tabulação dos dados foram calculados as médias e o desvio padrão, seguidos da análise com base na série histórica dos resultados encontrados no PARA.

Para o cálculo do total de amostras de alimentos analisadas, somou-se o total de amostras de cada alimento analisado no respectivo ano. Para o cálculo da média de ingredientes ativos analisados, somou-se o número destes ingredientes analisados em cada alimento naquele ano, e o total dividiu-se pelo número de diferentes alimentos que foram analisados no respectivo ano.

Para traçar o perfil de agrotóxicos detectados nos alimentos ao longo dos anos do PARA, e levando-se em consideração os dados disponíveis nos relatórios, optou-se por classificar os agrotóxicos em não autorizados para a cultura ou não autorizados no país, agrotóxicos detectados acima do LMR, agrotóxicos detectados abaixo do LMR e aqueles sem informação, devido às substâncias detectadas abaixo de $0,01 \mathrm{mg} / \mathrm{kg}$ trazidas no último relatório.

O formato do último relatório do PARA não classifica, em nenhuma das categorias, os ingredientes ativos detectados nas amostras de alimentos com quantidade de ingrediente ativo abaixo de $0,01 \mathrm{mg} / \mathrm{kg}$, pois relata que, em alguns países, este é o ponto de corte para considerar a significância regulatória, bem como considerar seus efeitos na saúde, e que a contaminação pode ser proveniente de contaminação cruzada na lavoura, como, por exemplo, contaminação ambiental ou pulverização aérea no plantio de culturas vizinhas 17 .

As amostras foram consideradas com irregularidades quando apresentaram ingredientes ativos acima do LMR estabelecido pela Anvisa, algum ingrediente ativo não autorizado para a cultura ou não autorizado no país ou, ainda, quando apresentaram ambas as situações.

Para o cálculo da média de amostras irregulares por alimento, somaram-se os valores encontrados nos anos em que o alimento foi analisado, e o total dividiu-se pelo número de anos em que foram analisados. Para a obtenção da média de amostras irregulares por ano de análise, somaram-se os valores destas amostras de todos os alimentos analisados naquele ano, e o total dividiu-se pelo número de diferentes alimentos que foram analisados no respectivo ano. 
A média de ingredientes ativos detectados por alimento foi calculada baseando-se na soma dos valores encontrados nos anos em que o alimento foi analisado, e o total dividiu-se pelo número de anos em que foram analisados. Para o cálculo da média por ano de análise, somaram-se os valores de ingredientes ativos detectados em todos os alimentos analisados naquele ano, e o total dividiu-se pelo número de diferentes alimentos que foram analisados no respectivo ano.

Para o cálculo da média de ingredientes ativos detectados que estavam dentro do LMR, calculou-se, inicialmente, o percentual destes ingredientes em relação ao número total de diferentes ingredientes ativos detectados no alimento no respectivo ano. Somaram-se, então, os percentuais dos ingredientes ativos dentro do LMR de cada ano, dividindo-se pela quantidade de anos em que o alimento foi analisado. Procedeu-se da mesma forma para a média dos ingredientes ativos acima do LMR e para aqueles classificados como não autorizados para a cultura ou não autorizados no país.

Por não ser uma pesquisa envolvendo seres humanos, mas sim uma pesquisa com fonte de dados secundários de origem pública, não enquadrando-se na Resolução no 466/2012 do Conselho Nacional de Saúde 21, que trata de pesquisas e testes em seres humanos, o projeto não foi submetido ao Comitê de Ética.

\section{Resultados}

Desde a implantação do PARA em âmbito nacional, em 2001, foram analisados 25 tipos diferentes de alimentos, porém, apenas alface e tomate foram avaliados em todos os anos. Ocorreu uma grande variação na quantidade de amostras analisadas ao longo do PARA, bem como na quantidade de alimentos analisados. As principais justificativas apresentadas nos relatórios para essas variações foram a deterioração dos produtos até o momento de análise pelos laboratórios, a disponibilidade do alimento para a coleta e a capacidade dos laboratórios para a realização das análises. Somando-se a quantidade de amostras de alimentos analisados pelo PARA, ao longo dos seus 15 anos de funcionamento, chega-se a um total de 31.449 amostras (Tabela 1).

Quanto ao número de diferentes ingredientes ativos que foi analisado nos alimentos, a maior média $(159,6)$ foi no ano de 2009, com uma variabilidade grande entre os alimentos, haja vista o desvio padrão de 69,9 no mesmo ano (Tabela 1 ).

Os alimentos que apresentaram as maiores médias de porcentuais de amostras irregulares foram: pimentão, abobrinha, morango, goiaba e uva. Já os alimentos com as menores médias foram: fubá de milho e farinha de mandioca. No ano de 2011 foi possível encontrar a maior média de amostras irregulares. Já 2005 foi o ano com a menor quantidade de amostras irregulares (Tabela 2).

O último relatório ainda traz a DRfA e demonstra que apenas 1,11\% das amostras monitoradas representou potencial risco agudo à saúde. Assim, os demais apresentam segurança alimentar aceitável quanto aos potenciais riscos de intoxicação aguda 17 .

\section{Tabela 1}

Tipos de alimentos, total de amostras e média de ingredientes ativos analisados pelo Programa de Análise de Resíduos de Agrotóxicos em Alimentos (PARA), no período de 2001 a 2015. Brasil, 2019.

\begin{tabular}{|c|c|c|c|c|c|c|c|c|c|c|c|c|}
\hline & $2001 / 2002$ & 2003 & 2004 & 2005 & 2006 & 2007 & 2008 & 2009 & 2010 & 2011 & 2012 & 2013 a 2015 \\
\hline Tipos de alimentos analisados & 9 & 9 & 9 & 8 & 6 & 9 & 17 & 20 & 18 & 9 & 13 & 25 \\
\hline $\begin{array}{l}\text { Total de amostras de alimentos } \\
\text { analisadas }\end{array}$ & 1.278 & 1.369 & 1.354 & 1.195 & 923 & 1.198 & 1.773 & 3.130 & 2.488 & 1.628 & 3.062 & 12.051 \\
\hline $\begin{array}{l}\text { Média de ingredientes ativos } \\
\text { analisados }\end{array}$ & 77,90 & 91,89 & 93,00 & 92,00 & 93,00 & 106,00 & * & 159,65 & 92,50 & 100,33 & 119,15 & 150,24 \\
\hline DP & 10,40 & 0,33 & 0,00 & 0,00 & 0,00 & 0,00 & * & 69,90 & 18,00 & 19,60 & 31,00 & 45,20 \\
\hline
\end{tabular}

DP: desvio padrão.

Fonte: elaboração própria, com base na Portaria no 03/1992 do Ministério da Saúde 18 e nos relatórios do PARA 17,35,36,37,38,39.

* O relatório de 2008 não deixa claro quantos ingredientes ativos foram analisados em cada alimento. 
Tabela 2

Frequência (\%) de amostras irregulares de alimentos para consumo humano, no período de 2001 a 2015 , com base no Programa de Análise de Resíduos de Agrotóxicos em Alimentos (PARA). Brasil, 2019.

\begin{tabular}{|c|c|c|c|c|c|c|c|c|c|c|c|c|c|c|}
\hline Alimento & $2001 / 2002$ & 2003 & 2004 & 2005 & 2006 & 2007 & 2008 & 2009 & 2010 & 2011 & 2012 & 2013 a 2015 & Média (\%) & DP \\
\hline Arroz & NA & NA & NA & NA & NA & NA & 4,41 & 27,20 & 7,40 & 16,00 & 10,00 & 4,15 & 11,53 & 8,83 \\
\hline Feijão & NA & NA & NA & NA & NA & NA & 2,92 & 3,00 & 6,50 & 6,00 & 7,30 & 7,19 & 5,49 & 2,12 \\
\hline Fubá de milho & NA & NA & NA & NA & NA & NA & NA & NA & NA & NA & 2,90 & 1,78 & 2,34 & 0,79 \\
\hline Farinha de trigo & NA & NA & NA & NA & NA & NA & NA & NA & NA & NA & NA & 7,50 & 7,50 & 0,00 \\
\hline Abacaxi & NA & NA & NA & NA & NA & NA & 9,47 & 44,10 & 32,80 & NA & 41,00 & 15,41 & 28,56 & 15,42 \\
\hline Banana & 6,53 & 2,22 & 3,59 & 3,14 & NA & 4,32 & 1,03 & 3,50 & NA & NA & NA & 2,79 & 3,39 & 1,61 \\
\hline Goiaba & NA & NA & NA & NA & NA & NA & NA & NA & NA & NA & NA & 45,56 & 45,56 & 0,00 \\
\hline Laranja & 1,41 & 0,00 & 4,91 & 4,71 & 0,00 & 6,04 & 14,85 & 10,30 & 12,20 & NA & 28,00 & 8,06 & 8,23 & 8,15 \\
\hline Maçã & 4,04 & 3,67 & 4,96 & 3,05 & 5,33 & 2,90 & 3,92 & 5,30 & 8,90 & NA & 8,00 & 10,60 & 5,52 & 2,54 \\
\hline Mamão & 19,50 & 37,56 & 2,50 & 0,00 & NA & 17,20 & 17,31 & 38,80 & 30,40 & 20,00 & NA & 17,45 & 20,11 & 12,96 \\
\hline Manga & NA & NA & NA & NA & NA & NA & 0,99 & 8,10 & 4,00 & NA & NA & 15,98 & 7,27 & 6,49 \\
\hline Morango & 46,03 & 54,55 & 39,07 & NA & 37,70 & 43,60 & 36,05 & 50,80 & 63,40 & NA & 59,00 & 72,61 & 50,33 & 12,09 \\
\hline Uva & NA & NA & NA & NA & NA & NA & 32,67 & 56,40 & NA & 27,00 & 29,00 & 74,55 & 43,93 & 20,78 \\
\hline Alface & 8,64 & 6,67 & 14,00 & 46,46 & 28,70 & 40,00 & 19,80 & 38,40 & 54,20 & 43,00 & 45,00 & 36,38 & 31,73 & 15,87 \\
\hline Couve & NA & NA & NA & NA & NA & NA & NA & NA & 44,20 & 31,90 & NA & 34,21 & 36,80 & 6,50 \\
\hline Repolho & NA & NA & NA & NA & NA & NA & 8,82 & 20,50 & 6,30 & NA & NA & 16,08 & 12,93 & 6,53 \\
\hline Abobrinha & NA & NA & NA & NA & NA & NA & NA & NA & NA & NA & 48,00 & 77,77 & 62,89 & 21,05 \\
\hline Pepino & NA & NA & NA & NA & NA & NA & NA & 54,80 & 57,40 & 44,00 & 42,00 & 29,77 & 45,59 & 11,06 \\
\hline Pimentão & NA & NA & NA & NA & NA & NA & 64,36 & 80,00 & 91,80 & 90,00 & NA & 88,88 & 83,01 & 11,35 \\
\hline Tomate & 26,10 & 0,00 & 7,36 & 4,38 & 2,01 & 44,70 & 18,27 & 32,60 & 16,30 & 12,00 & 16,00 & 32,05 & 17,65 & 13,84 \\
\hline Batata & 22,20 & 8,65 & 1,79 & 0,00 & 0,00 & 1,36 & 2,00 & 1,20 & 0,00 & NA & NA & 4,44 & 4,16 & 6,86 \\
\hline Beterraba & NA & NA & NA & NA & NA & NA & NA & 32,00 & 32,60 & NA & NA & 26,05 & 30,22 & 3,62 \\
\hline Cebola & NA & NA & NA & NA & NA & NA & 2,91 & 16,30 & 3,10 & NA & NA & 6,86 & 7,29 & 6,27 \\
\hline Cenoura & 0.00 & 0,00 & 19,54 & 11,90 & NA & 9,93 & 30,39 & 24,80 & 49,60 & 67,00 & 33,00 & 35,52 & 25,60 & 20,61 \\
\hline Farinha de mandioca & NA & NA & NA & NA & NA & NA & NA & NA & NA & NA & NA & 2,76 & 2,760 & 0,00 \\
\hline Média (\%) & 14,94 & 12,59 & 10,86 & 9,21 & 12,30 & 18,90 & 15,89 & 28,85 & 28,95 & 35,69 & 28,40 & 26,98 & & \\
\hline DP & 15,00 & 19,67 & 12,08 & 15,50 & 16,60 & 18,50 & 16,97 & 21,89 & 26,26 & 26,28 & 18,20 & 26,18 & & \\
\hline
\end{tabular}

DP: desvio padrão; NA: não analisado.

Fonte: elaboração própria, com base na Portaria no 03/1992 do Ministério da Saúde 18 e nos relatórios do PARA 17,35,36,37,38,39.

Além da quantidade de amostras irregulares detectadas pelo PARA, é importante, também, atentar para a quantidade de diferentes tipos de agrotóxicos encontrados nos alimentos, ao longo dos anos. A uva apresentou a maior média de diferentes ingredientes ativos detectados, seguida do pimentão, da couve, da abobrinha e do pepino. A cebola e a batata foram os alimentos com as menores médias de ingredientes ativos detectados. A cenoura, nos anos de 2001 a 2003, e a batata, em 2005, foram os únicos alimentos em que, nestas ocasiões, não foi detectado nenhum tipo de ingrediente ativo (Tabela 3).

Existe uma variabilidade grande no número de ingredientes ativos analisados e, consequentemente, detectados ao longo dos 15 anos do PARA, mas chamam a atenção alguns alimentos quando relaciona-se o número de detecções com a quantidade de ingredientes ativos analisados. A uva, por exemplo, no ano de 2015, foi um alimento com 51 diferentes ingredientes ativos detectados, de um total de apenas 73 que foram analisados neste alimento. Na goiaba, também no ano de 2015, foram analisados apenas 69 ingredientes ativos, dos quais 43 foram detectados nas amostras (Tabela 3).

Os anos de 2012 a 2015 registraram as maiores médias de diferentes ingredientes ativos detectados nos alimentos. Já a menor média de ingredientes ativos detectados foi obtida no ano de 2008 (Tabela 3). 


\section{Tabela 3}

Frequência de ingredientes ativos detectados nos alimentos para consumo humano, no período de 2001 a 2015 , com base no Programa de Análise de Resíduos de Agrotóxicos em Alimentos (PARA). Brasil, 2019.

\begin{tabular}{|c|c|c|c|c|c|c|c|c|c|c|c|c|c|c|}
\hline Alimento & $2001 / 2002$ & 2003 & 2004 & 2005 & 2006 & 2007 & 2008 & 2009 & 2010 & 2011 & 2012 & 2013 a 2015 & Média & DP \\
\hline Arroz & NA & NA & NA & NA & NA & NA & 4 & 30 & 12 & 14 & 8 & 33 & 16,83 & 11,91 \\
\hline Feijão & NA & NA & NA & NA & NA & NA & 3 & 19 & 19 & 13 & 24 & 45 & 20,50 & 14,00 \\
\hline Fubá de milho & NA & NA & NA & NA & NA & NA & NA & NA & NA & NA & 10 & 7 & 8,50 & 2,12 \\
\hline Farinha de trigo & NA & NA & NA & NA & NA & NA & NA & NA & NA & NA & NA & 17 & 17,00 & 0,00 \\
\hline Abacaxi & NA & NA & NA & NA & NA & NA & 4 & 20 & 19 & NA & 35 & 12 & 18,00 & 11,47 \\
\hline Banana & 2 & 5 & 5 & 5 & NA & 11 & 1 & 11 & NA & NA & NA & 23 & 7,88 & 7,12 \\
\hline Goiaba & NA & NA & NA & NA & NA & NA & NA & NA & NA & NA & NA & 43 & 43,00 & 0,00 \\
\hline Laranja & 5 & 4 & 18 & 24 & 7 & 19 & 7 & 20,00 & 32 & NA & 59 & 64 & 23,55 & 20,74 \\
\hline Maçã & 13 & 9 & 13 & 18 & 13 & 18 & 3 & 17 & 14 & NA & 22 & 47 & 17,00 & 11,15 \\
\hline Mamão & 11 & 10 & 3 & 2 & NA & 25 & 12 & 29 & 20 & 22 & NA & 49 & 18,30 & 14,06 \\
\hline Manga & NA & NA & NA & NA & NA & NA & 1 & 16 & 5 & NA & NA & 15 & 9,25 & 7,41 \\
\hline Morango & 15 & 23 & 17 & NA & 22 & 19 & 14 & 25 & 28 & NA & 47 & 48 & 25,80 & 12,25 \\
\hline Uva & NA & NA & NA & NA & NA & NA & 11 & 56 & NA & 33 & 27 & 51 & 35,60 & 18,30 \\
\hline Alface & 9 & 8 & 9 & 11 & 5 & 4 & 10 & 21 & 20 & 20 & 27 & 42 & 15,50 & 11,03 \\
\hline Couve & NA & NA & NA & NA & NA & NA & NA & 40 & 20 & NA & NA & 35 & 31,67 & 10,41 \\
\hline Repolho & NA & NA & NA & NA & NA & NA & 6 & 15 & 5 & NA & NA & 19 & 11,25 & 6,85 \\
\hline Abobrinha & NA & NA & NA & NA & NA & NA & NA & NA & NA & NA & 19 & 31 & 25,00 & 8,49 \\
\hline Pepino & NA & NA & NA & NA & NA & NA & NA & 31 & 17 & 17 & 22 & 38 & 25,00 & 9,25 \\
\hline Pimentão & NA & NA & NA & NA & NA & NA & 22 & 30 & 33 & 32 & NA & 59 & 35,20 & 13,99 \\
\hline Tomate & 15 & 11 & 16 & 17 & 5 & 18 & 9 & 26 & 28 & 27 & 36 & 63 & 22,58 & 15,54 \\
\hline Batata & 4 & 2 & 4 & 0 & 5 & 5 & 2 & 5 & 3 & NA & NA & 18 & 4,80 & 4,92 \\
\hline Beterraba & NA & NA & NA & NA & NA & NA & NA & 20 & 15 & NA & NA & 14 & 16,33 & 3,21 \\
\hline Cebola & NA & NA & NA & NA & NA & NA & 1 & 6 & 4 & NA & NA & 6 & 4,25 & 2,36 \\
\hline Cenoura & 0 & 0 & 3 & 5 & NA & 14 & 5 & 14 & 15 & 23 & 29 & 36 & 13,09 & 12,07 \\
\hline $\begin{array}{l}\text { Farinha de } \\
\text { mandioca }\end{array}$ & NA & NA & NA & NA & NA & NA & NA & NA & NA & NA & NA & 9 & 9,00 & 0,00 \\
\hline Média & 8,22 & 8,00 & 9,78 & 10,25 & 9,50 & 14,78 & 6,76 & 22,55 & 17,17 & 22,33 & 28,08 & 32,96 & 15,86 & \\
\hline DP & 5,67 & 6,75 & 6,30 & 8,64 & 6,86 & 6,96 & 5,67 & 11,74 & 9,24 & 7,24 & 13,94 & 18,01 & & \\
\hline
\end{tabular}

DP: desvio padrão; NA: não analisado.

Fonte: elaboração própria, com base na Portaria no 03/1992 do Ministério da Saúde 18 e nos relatórios do PARA 17,35,36,37,38,39.

A quantidade de agrotóxico detectada em cada amostra não foi apresentada em todos os relatórios do PARA, por isto, preferiu-se, nesta pesquisa, demonstrar quais os tipos de agrotóxicos detectados e se estavam dentro dos LMR definidos pela Anvisa.

Dos 25 alimentos já analisados pelo PARA, 14 deles registram médias acima de 50\% de ingredientes ativos não autorizados, entre aqueles que foram detectados. São eles: arroz, beterraba, couve, alface, cebola, abacaxi, manga, goiaba, morango, uva, abobrinha, repolho, farinha de mandioca e pimentão (Tabela 4).

No ano de 2008, foram obtidos os maiores percentuais de ingredientes ativos classificados como não autorizados. Alimentos como abacaxi, arroz, banana, laranja, manga, uva, repolho, cenoura e cebola apresentaram 100\% dos ingredientes ativos detectados classificados como não autorizados (Tabela 5).

A cebola, embora seja um dos alimentos citados com a menor média de diferentes ingredientes ativos detectados, registrou 73\% de classificados como não autorizados. Alguns alimentos, além de apresentarem os maiores porcentuais de amostras irregulares, demonstraram elevados números de 


\section{Tabela 4}

Análise dos ingredientes ativos detectados em alimentos para consumo humano, no período de 2001 a 2015 , com base no Programa de Análise de Resíduos de Agrotóxicos em Alimentos (PARA). Brasil, 2019.

\begin{tabular}{|c|c|c|c|c|c|c|c|c|}
\hline \multirow[t]{2}{*}{ Alimento } & \multicolumn{2}{|c|}{$\begin{array}{l}\text { Ingredientes ativos dentro } \\
\text { do LMR recomendado em } \\
\text { alimentos para consumo } \\
\text { humano }\end{array}$} & \multicolumn{2}{|c|}{$\begin{array}{l}\text { Ingredientes ativos acima } \\
\text { do LMR recomendado em } \\
\text { alimentos para consumo } \\
\text { humano }\end{array}$} & \multicolumn{2}{|c|}{$\begin{array}{c}\text { Ingredientes ativos não } \\
\text { autorizados para a cultura } \\
\text { ou não autorizados no } \\
\text { país }\end{array}$} & \multicolumn{2}{|c|}{$\begin{array}{l}\text { Ingredientes ativos } \\
\text { sem informação }\end{array}$} \\
\hline & Média (\%) & DP & Média (\%) & DP & Média (\%) & DP & Média (\%) & DP \\
\hline Arroz & 39,51 & 27,39 & 2,07 & 3,69 & 54,91 & 28,57 & 3,67 & 8,98 \\
\hline Feijão & 48,50 & 27,43 & 13,37 & 10,90 & 34,79 & 16,69 & 3,33 & 8,16 \\
\hline Fubá de milho & 71,00 & 1,01 & 17,00 & 4,04 & 12,00 & 3,03 & 0,00 & 0,00 \\
\hline Farinha de trigo & 64,70 & 0,00 & 5,88 & 0,00 & 23,53 & 0,00 & 5,00 & 0,00 \\
\hline Abacaxi & 16,00 & 11,20 & 13,00 & 14,90 & 71,00 & 20,00 & 0,00 & 0,00 \\
\hline Banana & 41,00 & 24,80 & 23,00 & 22,01 & 37,00 & 27,80 & 0,00 & 0,00 \\
\hline Goiaba & 6,98 & 0,00 & 4,65 & 0,00 & 46,51 & 0,00 & 42,00 & 0,00 \\
\hline Laranja & 67,00 & 26,16 & 5,00 & 6,25 & 26,00 & 22,55 & 1,00 & 5,00 \\
\hline Maçã & 61,02 & 22,98 & 10,00 & 10,29 & 27,73 & 15,80 & 0,40 & 1,20 \\
\hline Mamão & 40,83 & 18,41 & 24,71 & 19,83 & 33,43 & 20,95 & 1,00 & 3,16 \\
\hline Manga & 28,00 & 22,17 & 8,00 & 12,63 & 59,00 & 29,28 & 5,00 & 10,00 \\
\hline Morango & 16,32 & 8,50 & 15,68 & 6,89 & 67,00 & 7,10 & 1,00 & 4,00 \\
\hline Uva & 29,00 & 17,92 & 14,07 & 6,25 & 56,37 & 20,49 & 0,80 & 1,78 \\
\hline Alface & 18,03 & 10,63 & 5,00 & 5,97 & 76,70 & 11,57 & 0,25 & 0,86 \\
\hline Couve & 7,00 & 2,70 & 14,64 & 4,67 & 77,50 & 2,50 & 1,00 & 1,73 \\
\hline Repolho & 22,00 & 19,12 & 7,00 & 13,15 & 69,00 & 26,14 & 2,75 & 5,50 \\
\hline Abobrinha & 4,24 & 1,44 & 6,87 & 5,16 & 58,23 & 36,74 & 31,00 & 43,84 \\
\hline Pepino & 31,12 & 12,40 & 9,66 & 5,18 & 56,05 & 13,73 & 3,20 & 7,15 \\
\hline Pimentão & 15,00 & 8,63 & 10,06 & 4,80 & 72,49 & 6,77 & 2,00 & 5,00 \\
\hline Tomate & 59,80 & 24,65 & 14,00 & 11,84 & 25,47 & 18,46 & 1,00 & 3,00 \\
\hline Batata & 55,00 & 34,75 & 13,00 & 16,84 & 18,00 & 19,57 & 4,00 & 14,00 \\
\hline Beterraba & 16,58 & 4,27 & 2,00 & 3,84 & 81,00 & 3,37 & 0,00 & 0,00 \\
\hline Cebola & 23,00 & 15,77 & 0,00 & 0,00 & 73,00 & 20,83 & 4,00 & 8,50 \\
\hline Cenoura & 21,91 & 22,96 & 8,00 & 20,00 & 49,00 & 31,63 & 3,00 & 8,00 \\
\hline $\begin{array}{l}\text { Farinha de } \\
\text { mandioca }\end{array}$ & 11,11 & 0,00 & 0,00 & 0,00 & 66,66 & 0,00 & 22,00 & 0,00 \\
\hline
\end{tabular}

DP: desvio padrão; LMR: limite máximo de resíduos.

Fonte: elaboração própria, com base na Portaria no 03/1992 do Ministério da Saúde 18 e nos relatórios do PARA 17,35,36,37,38,39.

ingredientes ativos detectados, sendo, também, campeões das médias de ingredientes ativos não autorizados, como é o caso do morango, da uva, da abobrinha e do pimentão (Tabela 4).

É preciso, porém, observar com mais cuidado alguns elementos apresentados nos relatórios referentes ao dado isolado de irregularidade, pois, a exemplo da laranja e da maçã, alimentos que, ao longo da maioria dos anos em que foram analisados apresentaram irregularidades em menos de 30\% das amostras (Tabela 2) e maioria dos agrotóxicos dentro do LMR (Tabela 4), são frutas nas quais detectou-se grande número de diferentes agrotóxicos em 2015 (Tabela 3).

Na presente pesquisa, percebeu-se, também, que os LMR de 72 ingredientes ativos analisados pelo PARA foram revistos e alterados ao longo dos anos, sendo que grande parte deles tornou aceitável uma maior quantidade de resíduos. Como exemplos dessas situações, podem ser citados o LMR da azoxistrobina que, na laranja, passou de não autorizada para um LMR de $0,5 \mathrm{mg} / \mathrm{kg}$, e a alface, que foi de um LMR de 0,5 para $1 \mathrm{mg} / \mathrm{kg}$. A bifentrina, substância antes classificada como não autorizada, passou a ter um LMR em quatro alimentos: banana, laranja, mamão e tomate. Outras alterações 
Tabela 5

Análise dos ingredientes ativos, por ano, detectados em alimentos para consumo humano, no período de 2001 a 2015 , com base no Programa de Análise de Resíduos de Agrotóxicos em Alimentos (PARA). Brasil, 2019.

\begin{tabular}{|c|c|c|c|c|c|c|c|c|}
\hline \multirow[t]{2}{*}{ Ano } & \multicolumn{2}{|c|}{$\begin{array}{c}\text { Ingredientes ativos dentro } \\
\text { do LMR recomendado em } \\
\text { alimentos para consumo } \\
\text { humano }\end{array}$} & \multicolumn{2}{|c|}{$\begin{array}{l}\text { Ingredientes ativos acima } \\
\text { do LMR recomendado em } \\
\text { alimentos para consumo } \\
\text { humano }\end{array}$} & \multicolumn{2}{|c|}{$\begin{array}{c}\text { Ingredientes ativos não } \\
\text { autorizados para a cultura } \\
\text { ou não autorizados no } \\
\text { país }\end{array}$} & \multicolumn{2}{|c|}{$\begin{array}{l}\text { Ingredientes ativos sem } \\
\text { informação }\end{array}$} \\
\hline & Média (\%) & DP & Média (\%) & DP & Média (\%) & DP & Média (\%) & DP \\
\hline $2001 / 2002$ & 40,32 & 28,12 & 14,42 & 14,97 & 35,24 & 23,80 & 0,00 & 0,00 \\
\hline 2003 & 45,52 & 32,69 & 11,47 & 19,01 & 31,88 & 28,07 & 0,00 & 0,00 \\
\hline 2004 & 46,11 & 25,22 & 22,50 & 26,56 & 31,37 & 22,63 & 0,00 & 0,00 \\
\hline 2005 & 38,60 & 32,07 & 17,35 & 21,12 & 31,53 & 29,97 & 0,00 & 0,00 \\
\hline 2006 & 57,38 & 31,47 & 10,74 & 9,23 & 30,59 & 34,84 & 0,00 & 0,00 \\
\hline 2007 & 57,37 & 31,46 & 7,30 & 10,16 & 37,31 & 28,22 & 0,00 & 0,00 \\
\hline 2008 & 0,00 & 0,00 & 13,83 & 16,10 & 86,16 & 16,10 & 0,00 & 0,00 \\
\hline 2009 & 42,33 & 21,74 & 7,58 & 7,22 & 50,06 & 21,11 & 0,00 & 0,00 \\
\hline 2010 & 40,99 & 27,00 & 6,95 & 6,46 & 52,04 & 25,90 & 0,00 & 0,00 \\
\hline 2011 & 39,24 & 23,05 & 8,08 & 7,60 & 52,68 & 21,37 & 0,00 & 0,00 \\
\hline 2012 & 40,74 & 24,13 & 9,81 & 7,79 & 49,42 & 25,45 & 0,00 & 0,00 \\
\hline 2013 a 2015 & 32,04 & 19,47 & 11,55 & 9,42 & 41,17 & 19,86 & 15,28 & 15,42 \\
\hline
\end{tabular}

DP: desvio padrão; LMR: limite máximo de resíduos.

Fonte: elaboração própria, com base na Portaria no 03/1992 do Ministério da Saúde 18 e nos relatórios do PARA 17,35,36,37,38,39.

chamam ainda mais a atenção devido à sua amplitude, por exemplo: o clorotalonil, antes também não autorizado, passou a ser usado na banana com um LMR de $3 \mathrm{mg} / \mathrm{kg}$; a alface, com um LMR de $6 \mathrm{mg} / \mathrm{kg}$; e o pimentão, com um LMR que passou de 0,1 para $5 \mathrm{mg} / \mathrm{kg}$.

Além dos resultados apresentados, alguns relatórios trouxeram, ainda, informações sobre a presença de mais de um ingrediente ativo detectado em uma mesma amostra de alimento. Ao longo dos anos do PARA, destacou-se o pimentão, no qual, em uma mesma amostra, foram detectados oito diferentes tipos de ingredientes ativos no ano de 2011. Como justificativa para o aparecimento de mais de um ingrediente ativo em uma mesma amostra de alimento, o último relatório do PARA mostra a necessidade de aplicação de ingredientes ativos com finalidades diferentes, formulações que têm mais de um ingrediente ativo, contato entre os alimentos, resíduos do solo etc.

Ao longo dos 15 anos do PARA, há uma persistência na detecção de ingredientes ativos não autorizados para a cultura ou não autorizados no país, sendo que, a partir do ano de 2007 , ocorreu um aumento deste percentual, chegando, em 2008, próximo aos 90\% dos ingredientes ativos detectados (Tabela 5).

\section{Discussão}

Entre os desafios apresentados pelo PARA, podem ser citados a variação na periodicidade de coleta e avaliação, variação dos alimentos e ingredientes ativos analisados, bem como o formato de seus relatórios, o que dificulta qualquer comparação no decorrer dos anos do PARA, uma vez que, quanto mais amostras e ingredientes ativos são analisados, aumenta a probabilidade de serem detectados os agrotóxicos.

A redução de amostras irregulares, a partir de 2012 (Tabela 2), bem como a redução da média de ingredientes ativos classificados como dentro do LMR e acima do LMR (Tabela 5), podem ter sofrido influências tanto da nova classificação incluída no último relatório, daqueles ingredientes ativos abaixo de $0,01 \mathrm{mg} / \mathrm{kg}$ que não entraram em nenhuma categoria, como da alteração dos LMR, que podem 
ter reclassificado as amostras, antes irregulares, agora como regulares. Porém, não se pode descartar, também, que a redução no número de amostras irregulares pode ter sido influenciada pelo próprio ponto forte do PARA, o de disparar na sociedade a discussão e fortalecer grupos e fóruns sociais no controle dos agrotóxicos nos alimentos no Brasil. Como exemplo, tem-se a criação dos fóruns nacional e estaduais para controle e combate ao uso de agrotóxicos e a Campanha Permanente Contra os Agrotóxicos e pela Vida 17.

O PARA proporcionou muitas reflexões sobre a regulação de resíduos de agrotóxicos nos alimentos e são de extrema importância o seu aperfeiçoamento e a sua continuidade. Entre os avanços, é visível que, ao longo dos anos, ocorreu aumento no número de amostras analisadas, aumento no número de ingredientes ativos analisados e novos alimentos foram incluídos para análise, o que foi possível a partir da ampliação da rede de laboratórios contratada para dar suporte ao PARA.

O resultado apresentado pelo PARA é capaz de subsidiar as ações de fiscalização da vigilância sanitária e dar suporte a uma estimativa de exposição alimentar aos agrotóxicos, o que é imprescindível na reavaliação dos ingredientes ativos já registrados, e fornece informações ao Ministério da Agricultura para orientar as ações de fiscalização no campo. Outro avanço proporcionado pelos resultados do PARA foi a criação de legislações específicas para o controle e a fiscalização da rastreabilidade de hortifrutis em toda a cadeia produtiva desses alimentos, o que possibilita aos fiscais mapear e penalizar os irresponsáveis em casos de amostras irregulares 17.

Para que se possa aperfeiçoar ainda mais o PARA e garantir a sua continuidade, à luz dos seus objetivos apresentados em seu primeiro relatório, no ano de 2001, é preciso ampliar ainda mais o escopo dos diferentes tipos de alimentos analisados, a fim de informar a real exposição alimentar baseada em todos os alimentos mais consumidos pelos brasileiros. Da mesma forma, é necessário ampliar o número de ingredientes ativos analisados. Alguns alimentos, como o leite e seus derivados, diversos tipos de carnes e ovos, entre outros, presentes no cotidiano dos brasileiros 22, não são avaliados pelo PARA, mas também podem estar contaminados por agrotóxicos.

Outro desafio, ainda, para o alcance da segurança alimentar, citada como um dos objetivos do PARA, é aprofundar a discussão a respeito do conceito e da classificação de risco agudo e crônico empregado para estabelecer se a amostra está satisfatória ou insatisfatória. O último relatório traz o conceito de DRfA e, com esta, classifica apenas 1,11\% das amostras monitoradas com potencial risco agudo à saúde. Esse tipo de critério usado para caracterizar a amostra como insatisfatória pode induzir à banalização ou ao ocultamento do risco crônico presente na exposição alimentar aos agrotóxicos.

Enquanto no Brasil, o máximo de diferentes tipos de alimentos analisados foi 25 , sem padronização de número de amostras e ingredientes ativos, a União Europeia analisa mais de 60 tipos, incluindo alimentos processados, leite, alimentos para bebês e até mesmo ração para animais. Além disso, os países europeus padronizam o mínimo de ingredientes ativos que devem ser pesquisados todos os anos. No total, foram pesquisados mais de 680 ingredientes ativos no ano de 201323 , chegando a 774 deles no ano de 2015 24. No Brasil, o máximo de ingredientes ativos pesquisados foi 228, de um total de mais de 500 registrados no país 25 . Logo, o diagnóstico apresentado pelo PARA não reflete a real exposição alimentar aos agrotóxicos.

Existem diferenças, também, relativas à quantidade de amostras de alimentos analisadas entre o Brasil e os países europeus. Enquanto no Brasil, durante os 15 anos do PARA, foram analisadas 31.449 amostras, somente no ano de 2015 a União Europeia analisou quase 85 mil amostras.

Um dos últimos dados da União Europeia de 2013 mostra também que, mesmo analisando mais ingredientes ativos, em mais amostras e com os seus LMR bem mais rigorosos do que os utilizados no Brasil, o PARA, nesse ano, detectou irregularidades em quase 26\% das amostras, e na Europa este valor foi inferior a $3 \% 23$.

É preciso, porém, aprofundar o debate sobre os parâmetros usados pelo PARA para apresentar o diagnóstico descrito anteriormente, pois estes critérios interferem diretamente nos seus resultados. Os LMR são contabilizados para as culturas para as quais são autorizados os diversos agrotóxicos. No caso do Brasil, os resultados apresentados pelo PARA demonstram que diversos ingredientes ativos são utilizados em outras culturas para as quais não são autorizados, o que aumenta a exposição a tais substâncias, não previstas na IDA. O fato de o perfil de irregularidades prevalentes nos alimentos ser de ingredientes ativos não autorizados para a cultura, ou não autorizados no país, traz uma preocu- 
pação ainda maior quanto ao risco existente, por estas substâncias não estarem incluídas nos cálculos da IDA.

O próprio procedimento utilizado para o cálculo da IDA também é bastante questionável, pela fragilidade das evidências científicas que o sustentam. Inicialmente, são administradas a cobaias, por via oral, doses crescentes do ingrediente ativo buscando estabelecer a dose limite, que não cause efeitos observáveis nos animais [no observable effect level - NOAEL]. Uma vez estabelecida, essa dose é dividida por 100 devido às diferenças interespécies e intraespécies, definida, então, como aceitável para os humanos 26. Porém, essa IDA, para humanos, desconsidera os efeitos sinérgicos de outras drogas ou outros fatores intervenientes do próprio agrotóxico ou da sua ação sinérgica a outros produtos químicos 27 .

Os dois tipos de cálculos, que são aplicados ao objeto de estudo da toxicologia, fazem parte de uma retórica da ocultação, que é útil apenas para transmitir a ideia de confiança nos chamados limites de tolerância. É questionada também a avaliação de risco para a definição daqueles limites por desconsiderar fatores importantes, como: a ação simultânea de diversos compostos químicos - entre eles, os diversos tipos de agrotóxicos usados no país - agindo simultaneamente no corpo humano; as diferenças de suscetibilidade, conforme a idade e os fatores genéticos; e diferentes fontes de exposição e os efeitos crônicos dela 27.

Quando comparados os LMR adotados para algumas substâncias no Brasil com aqueles adotados pelos países europeus também são observadas diferenças, em alguns casos, bastante significativas. Ou seja, ao serem adotados os parâmetros europeus, o diagnóstico apresentado pelo PARA poderia ser bem mais preocupante do que já é. Estudos comparando os programas de controle do uso de agrotóxicos na União Europeia e no Brasil listam alguns dos principais ingredientes ativos que aparecem com frequência nos alimentos brasileiros, cujos LMR mostram diferenças significativas em relação aos dos países da Europa. Exemplos disso são: a abamectina, na uva, com um LMR 30 vezes maior no Brasil; o acetamiprimido, na batata, com um LMR 50 vezes maior; a malationa, no feijão, com um LMR 400 vezes maior 24; e o glifosato, na soja, com um LMR 200 vezes maior 28.

Existem também diferenças entre alguns LMR de agrotóxicos permitidos na água dos brasileiros e dos europeus. O clorpirifós, muito encontrado também nos alimentos, tem um LMR 300 vezes maior no Brasil do que na União Europeia; o tebuconazol, um LMR 1.800 vezes maior; e o glifosato, com um valor chegando a ser 5 mil vezes maior no Brasil do que na Europa 28.

Além disso, a oscilação detectada nos valores dos LMR de alguns ingredientes ativos para alguns alimentos influencia diretamente o resultado do PARA, quando amostras anteriormente classificadas como não satisfatórias passam, agora, a ser consideradas sem irregularidades.

É preciso lembrar a posição do Brasil como economia periférica e sua dependência econômica diante dos países centrais nas relações econômicas mundiais, os quais lhe impõem uma atuação intensamente subordinada às necessidades de reprodução do capital internacional. Na prática, isso se reflete em políticas fiscais permissivas, que possibilitam a entrada de empresas produtoras de agrotóxicos em solo brasileiro, além de mais facilidades na concessão de créditos aos produtores que utilizam agrotóxicos 29 . Aqui, as tecnologias apresentadas pela indústria do campo e impostas às economias periféricas, para a extração da mais-valia, encontram um ambiente favorável para a degradação da terra e dos recursos naturais, bem como para a superexploração dos trabalhadores. Infelizmente, fazem parte desse pacote a insegurança alimentar, a insustentabilidade ambiental e os impactos negativos à saúde humana 30 .

Neumann et al. 31 inserem, no debate, a contradição existente entre o interesse coletivo e o individual quando, no Congresso Nacional - que define as legislações referentes ao uso e controle de agrotóxicos no país - existe uma bancada ruralista, que interfere diretamente nessas decisões.

Levando-se em consideração a alimentação adequada e saudável como um direito humano, e a obrigação do Estado brasileiro de garantir a segurança alimentar e nutricional aos seus cidadãos, estabelecida na Constituição Federal, é necessário, também, ampliar o debate com a sociedade e a transparência de informações, incluindo os estudos toxicológicos realizados e os critérios utilizados para a autorização de novos agrotóxicos no Brasil, haja vista a ampliação de novas substâncias aprovadas, principalmente sob o novo governo, a partir do ano de 2019.

Em paralelo à agenda de maior liberação de agrotóxicos no país, observa-se maior desmobilização da sociedade civil organizada. No que concerne ao controle do uso de agrotóxicos, um estudo realiza- 
do por Soares et al. 32 revela o quanto ainda é frágil a discussão deste tema tão relevante para a sociedade, inclusive entre representantes e órgãos vinculados à saúde. Os pesquisadores, ao entrevistarem conselheiros de saúde, descobriram durante as entrevistas que a temática dos agrotóxicos foi inédita naquele espaço, e que os entrevistados relataram falta de informações sobre o tema, o que dificulta uma atuação mais crítica, bem como a percepção de que são sujeitos de transformação da realidade.

A desmobilização da sociedade civil, promovida pelo atual governo brasileiro, também dificulta muito o acesso à informação e a articulação de medidas no controle do uso de agrotóxicos no país, a exemplo da extinção do Conselho Nacional de Segurança Alimentar e Nutricional (CONSEA), a partir de uma Medida Provisória editada pelo atual presidente Jair Bolsonaro. Tal medida não somente compromete as ações de garantia do Direito Humano à Alimentação Adequada, como também constitui evidente retrocesso social quando desmonta um espaço de participação social, haja vista que $2 / 3$ dos membros do CONSEA eram da sociedade civil 33 .

Na contramão da agenda liberal do governo, o novo Guia Alimentar para a População Brasileira, lançado em 2014, além da regra de ouro para uma alimentação saudável, de preferir alimentos in natura ou minimamente processados, preocupa-se e amplia o debate para que estes alimentos derivem de um sistema alimentar social e ambientalmente sustentável 34.

O PARA é um instrumento importante que avançou muito ao longo dos anos. O PARA dá visibilidade ao uso abusivo de agrotóxicos no Brasil, mesmo ainda não apresentando o real diagnóstico de resíduos de agrotóxicos nos alimentos, pelas limitações já apresentadas. Embora os LMR utilizados como parâmetros para a apresentação das irregularidades encontradas nos alimentos tenham diversas limitações, não deixam de constituir uma barreira à livre utilização dos venenos, sem a qual a situação poderia ser ainda pior.

Novos estudos são necessários para a compreensão da permanência ou do aumento da presença de agrotóxicos, autorizados ou não, nos alimentos comercializados no país, diante da farta evidência dos prejuízos que acarretam à saúde. A reflexão sobre a determinação social do uso de agrotóxicos no Brasil, incluindo os interesses econômicos em tais condições, parece fundamental para elucidar a gênese dos problemas de regulação destas substâncias nos alimentos.

Entre os pontos fortes deste estudo, destacam-se a demonstração da existência do monitoramento e a identificação da presença de resíduos de agrotóxicos proibidos no país, para a cultura ou acima dos limites permitidos, possibilitando um alerta ainda maior para a sociedade civil organizada. Além disso, o presente artigo contribui de forma significativa para a avaliação e o aprimoramento do PARA. Entre os pontos fracos, está a impossibilidade de verificar adequadamente a quantidade dos resíduos nos diversos tipos de alimentos devido à inconstância de suas pesquisas, à variação dos alimentos analisados e às mudanças nos limites considerados aceitáveis. 


\section{Colaboradores}

C. V. A. Lopes e G. S. C. Albuquerque participaram da elaboração do projeto, análise e interpretação dos dados, além da redação do artigo e aprovação final da versão a ser publicada.

\section{Informações adicionais}

ORCID: Carla Vanessa Alves Lopes (0000-00027157-4285); Guilherme Souza Cavalcanti de Albuquerque (0000-0002-7544-412X).

\section{Conflito de interesses}

Os autores declaram não haver qualquer conflito de interesses nesta pesquisa e que ela não obteve qualquer fonte de financiamento.

\section{Agradecimentos}

Agradecemos a todos que, de alguma forma, contribuíram no andamento desta pesquisa. O presente trabalho é fruto da Dissertação de Mestrado apresentada ao Programa de Pós-graduação em Saúde Coletiva da Universidade Federal do Paraná.

\section{Referências}

1. Rigotto RM, Vasconcelos DP, Rocha MM. Pesticide use in Brazil and problems for public health. Cad Saúde Pública 2014; 30:1360-2.

2. Tambellini AT, Miranda AC. Saúde e ambiente. In: Giovanella L, Escorel S, Lobato LVC, Noronha JC, Carvalho AI, organizadores. Políticas e sistema de saúde no Brasil. 2a Ed. Rio de Janeiro: Editora Fiocruz; 2012. p. 1037-73.

3. Ruiz de Arcaute C, Costa CS, Demetrio PM, Natale GS, Ronco AE. Influence of existing site contamination on sensitivity of Rhinella fernandezae (Anura, Bufonidae) tadpoles to Lorsban $48 \mathrm{E}$ formulation of chlorpyrifos. Ecotoxicology 2012; 21:2338-48.

4. Egler M, Buss DF, Moreira JC, Baptista DF. Influence of agricultural land-use and pesticides on benthic macroinvertebrate assemblages in an agricultural river basin in southeast Brazil. Braz J Biol 2012; 72:437-43.

5. Tomé HVV, Barbosa WF, Martins GF, Guedes RN. Spinosad in the native stingless bee Melipona quadrifasciata: regrettable non-target toxicity of a bioinsecticide. Chemosphere 2015; 124:103-9.

6. Machado da Rosa J, Aroli CJ, Nunes-Silva P, Garcia FRM. Desaparecimento de abelhas polinizadoras nos sistemas naturais e agrícolas: existe uma explicação? Revista de Ciências Agroveterinárias 2019; 18:154-62.

7. Santana VS, Moura MCP, Nogueira FF. Mortalidade por intoxicação ocupacional relacionada a agrotóxicos, 2000-2009, Brasil. Rev Saúde Pública 2013; 47:598-606.

8. Ferreira de Siqueira D, de Moura RM, Laurentino GEC, de Araújo AJ, Cruz SL. Análise da exposição de trabalhadores rurais a agrotóxicos. Rev Bras Promoç Saúde 2013; 26:182-91.

9. Segatto MM, Ronamigo RR, Hohmann CB, Muller KR, Bakos L, Mastroeni S, et al. Residential and occupational exposure to pesticides may increase risk for cutaneous melanoma: a case-control study conducted in the south of Brazil. Int J Dermatol 2015; 54:527-38.
10. Silva AC, Camponogara S, Viero CM, Menegat RP, Dias GL, Miorin JD. Socioeconomic profile of rural workers cancer sufferers. Revista da Fundação Care 2016; 8:4891-97.

11. Murakami Y, Pinto NC, Albuquerque GSC, Perna PO, Lacerda A. Intoxicação crônica por agrotóxicos em fumicultores. Saúde Debate 2017; 41:563-76.

12. Lopes CVA, Albuquerque GSC. Agrotóxicos e seus impactos na saúde humana e ambiental. Saúde Debate 2018; 42:518-34.

13. Jardim ANO, Mello DC, Goes FCS, Frota Junior EF, Caldas ED. Pesticide residues in cashew apple, guava, kaki and peach: GC- $\mu \mathrm{ECD}$, GC-FPD and LC-MS/MS multiresidue method validation, analysis and cumulative acute risk assessment. Food Chem 2014; 164:195204.

14. Ciscato CHP, Gebara AB. Avaliação de resíduos de pesticidas na dieta brasileira, período de 2001 a 2010. Higiene Alimentar 2017; 31:110-4.

15. Lima, GPP, Teixeira da Silva JA, Bernhard AB, Pirozzi DCZ, Fleuri LF, Vianello F. Organic and conventional fertilisation procedures on the nitrate, antioxidants and pesticide content in parts of vegetables. Food Addit Contam Part B Surveill 2012; 5:188-93.

16. Selmi GFR, Trapé AZ. Proteção da saúde de trabalhadores rurais: a necessidade de padronização das metodologias de quantificação da exposição dérmica a agrotóxicos. Cad Saúde Pública 2014; 30:952-60.

17. Agência Nacional de Vigilância Sanitária. Programa de Análise de Resíduos de Agrotóxicos em Alimentos (PARA). Relatório de atividades 2013-2015. http://portal.anvisa.gov.br/ documents/111215/0/Relat\%C3\%B3rio+PARA+2013-2015_VERS\%C3\%83O-FINAL.pd f/494cd7c5-5408-4e6a-b0e5-5098cbf759f8 (acessado em 02/Mai/2017). 
18. Ministério da Saúde. Portaria no 03/MS/SVS, de 16 de janeiro de 1992. Diário Oficial da União 1992; 17 jan.

19. Agência Nacional de Vigilância Sanitária. Programa de Análise de Resíduos de Agrotóxicos em Alimentos (PARA). Relatório de atividades 2011-2012. http://portal.anvisa.gov.br/docu ments/111215/117818/Relat\%25C3\%25B3rio $\% 252$ B PARA \% 252 B $2011-12 \% 252$ B -\%252B30_10_13_1.pdf/d5e91 ef0 - 42354872-b180-99610507d8d5 (acessado em 30/ Mai/2017).

20. Agência Nacional de Vigilância Sanitária. Programa de Análise de Resíduos de Agrotóxicos em Alimentos (PARA). Relatório de atividades 2001-2007. http://portal. anvisa.gov.br/documents/111215/117818/ relatorio\%2B2001\%2B2007.pdf/460433e63d66-400b-8e93-48413ea8203f (acessado em 30/Mai/2017).

21. Conselho Nacional de Saúde. Resolução no 466, de 12 de dezembro de 2012. Diário Oficial da União 2013; 13 jun.

22. Instituto Brasileiro de Geografia e Estatística. Pesquisa de Orçamentos Familiares 20082009: análise do consumo alimentar pessoal no Brasil. Rio de Janeiro: Instituto Brasileiro de Geografia e Estatística; 2011.

23. Gonçalves MS. Uso sustentável de pesticidas: análise comparativa entre a União Europeia e o Brasil [Tese de Doutorado]. Lisboa: Programa de Doutoral em Alterações Climáticas e Políticas de Desenvolvimento Sustentável, Universidade de Lisboa; 2016.

24. European Food Safety Authority. The 2015 European Union report on pesticide residues in food. EFSA J 2017; 15(4). https://efsa.on linelibrary.wiley.com/doi/epdf/10.2903/j. efsa.2017.4791.

25. Agência Nacional de Vigilância Sanitária. Lista de ingredientes ativos com uso autorizado e banidos do Brasil. http://portal.anvisa.gov.br/ rss/-/asset_publisher/Zk4q6UQCj9Pn/con tent/id/3197746 (acessado em 10/Mai/2018).

26. Klaassen CD, Watkins III JB, organizadores. Fundamentos em toxicologia de Casarett e Doull. 2a Ed. Porto Alegre: Artmed; 2012.

27. Carneiro FF, Rigotto RM, Augusto LGS, Friedrich K, Búrigo AC, organizadores. Dossiê ABRASCO: um alerta sobre os impactos dos agrotóxicos na saúde. Rio de Janeiro: Escola Politécnica de Saúde Joaquim Venâncio, Fundação Oswaldo Cruz/Expressão Popular; 2015.

28. Bombardi LM. Geografia do uso de agrotóxicos no Brasil e conexões com a União Europeia. São Paulo: Faculdade de Filosofia, Letras e Ciências Humanas, Universidade de São Paulo; 2017.

29. Xavier GL. Agronegócio e capitalismo dependente na América Latina: o caso brasileiro. Argumentum 2017; 9:147-60.

30. Horii AKD. Contradições do capitalismo no campo: o uso de agrotóxicos e os desafios à saúde humana. Ciência Geográfica 2015; 19:190-201.
31. Neumann E, Fajardo S, Marin MZ. As transformações recentes no espaço rural brasileiro: análise do papel do Estado nas políticas de desenvolvimento rural nas décadas de 1970 a 1990. Revista Ra'e Ga 2017; 40:191-208.

32. Soares MMA, Zuchi AP, Lopes CVA, Anjos MCR. Percepção de conselheiros de saúde acerca do tema agrotóxicos: o papel da participação social em uma sociedade que adoece. Saúde Soc 2019; 28:337-49.

33. Castro IRR. A extinção do Conselho Nacional de Segurança Alimentar e Nutricional e a agenda de alimentação e nutrição. Cad Saúde Pública 2019; 35:e00009919.

34. Martinelli SS, Cavalli SB. Alimentação saudável e sustentável: uma revisão narrativa sobre desafios e perspectivas. Ciênc Saúde Colet 2019; 24:4251-61.

35. Agência Nacional de Vigilância Sanitária. Programa de Análise de Resíduos de Agrotóxicos em Alimentos (PARA). Relatório de atividades 2008. http://portal.anvisa.gov.br/ documents/111215/117818/nota\%2Btecni ca\%2B-\%2Bresultados\%2Bpara\%2B2008.pd f/78967b71-4df4-4b47-b5b3-6d71de54b392 (acessado em 30/Mai/2017).

36. Agência Nacional de Vigilância Sanitária. Programa de Análise de Resíduos de Agrotóxicos em Alimentos (PARA). Relatório de atividades 2009. http://portal.anvisa.gov. br/documents/111215/446371/Progra $\mathrm{ma}+\mathrm{de}+\mathrm{An} \% \mathrm{C} 3 \% \mathrm{~A} 1 \mathrm{lise}+\mathrm{de}+\mathrm{Res} \% \mathrm{C} 3 \% \mathrm{AD}$ duos+de+Agrot\%C3\%B3xicos+-+Relat\% C3\%B3rio+2009/8a6a6722-f009-442e-9760$569 b c 5279$ c8d (acessado em 30/Mai/2017).

37. Agência Nacional de Vigilância Sanitária. Programa de Análise de Resíduos de Agrotóxicos em Alimentos (PARA). Relatório de atividades 2010. http://portal.anvisa.gov. br/documents/111215/117818/Relat\% 25C3\%25B3rio\%2BPARA\%2B2010\%2B-\%2B Vers\%25C3\%25A3o\%2BFinal.pdf/f568427bc518-4a68-85b9-dd7680e55e07 (acessado em 30/Mai/2017).

38. Agência Nacional de Vigilância Sanitária. Programa de Análise de Resíduos de Agrotóxicos em Alimentos (PARA). Relatório de atividades 2011-2012. http://portal. avisa.gov.br/documents/111215/117818/ Relat\%2 5 C $3 \% 25$ B 3 rio\% 252 B PARA \%252B2011-12\%252B-\%252B30_10_13_1. pdf / d 5 e 91 ef $0-4235-4872$ - b 180 99610507d8d5 (acessado em 30/Mai/2017).

39. Agência Nacional de Vigilância Sanitária. Programa de Análise de Resíduos de Agrotóxicos em Alimentos (PARA). Relatório complementar relativo à segunda etapa das análises de amostras coletadas em 2012. http://portal. anvisa.gov.br/documents/111215/446371/ Programa+de+An\%C3\%A1lise+de+Res\% C3\%ADduos+de+Agrot\%C3\%B3xicos+-+Re lat\%C3\%B3rio+2009/8a6a6722-f009-442e9760-569bc5279c8d (acessado em 30/ Mai/2017). 


\section{Abstract}

In Brazil, since 2001, pesticides in food products have been monitored by the Program for Analysis of Pesticide Residues in Food Products (PARA, in Portuguese). The study aimed to analyze the challenges and strides in the control of pesticide residues in food products in Brazil based on the program's results from 2001 to 2015. A descriptive-analytical document review was conducted that used data from reports by the PARA, available at the website of the Brazilian Health Regulatory Agency. The program's main challenges feature the wide variation in the number of samples and active ingredients analyzed, as well as the reports' format. There is a need for more in-depth discussions of the criteria used for classification and authorization of active ingredients in Brazil, as well as the information's transparency to society. A total of 25 types of foods were analyzed, with more than 30,000 samples, since the PARA has been in force, but the maximum number of active ingredients analyzed was 228. The presence of active ingredients unauthorized for crops and unauthorized in the country was the principal cause of irregularities diagnosed in the foods. Although the program still faces various challenges, it helps spawn debate on the issue in society. Given the current government context of greater flexibility in the authorization of active ingredients in Brazil, it is necessary to strengthen the PARA.

Agrochemicals; Food and Nutrition Security; Health Surveillance

\section{Resumen}

En Brasil, desde 2001, la supervisión de la presencia de pesticidas en los alimentos se produce mediante el Programa de Análisis de Residuos de Pesticidas en Alimentos (PARA). El objetivo fue analizar los desafios y avances en el control de residuos de pesticidas en los alimentos, en Brasil, a partir de los resultados del PARA, entre los años 2001 y 2015. Investigación descriptivo-analítica documental, que utilizó datos provenientes de los informes del PARA, disponibles en la página web de la Agencia Nacional de Vigilancia Sanitaria. Entre los principales desafíos presentados por el PARA, están la gran variabilidad en el número de muestras e ingredientes activos analizados, así como en el formato de los informes presentados. Existe la necesidad de profundizar en las discusiones, respecto a los criterios utilizados para la clasificación $y$ autorización de los ingredientes activos en Brasil, así como la transparencia de esta información para la sociedad. En total, se analizaron 25 tipos de alimentos y más de 30 mil muestras, a lo largo de la vigencia del PARA, no obstante, el máximo de ingredientes activos analizados fue 228. La presencia de ingredientes activos no autorizados para el cultivo y no autorizados en el país fue la principal causa de irregularidades diagnosticadas en los alimentos. A pesar de que todavía existan diversos desafios presentados en el PARA, contribuye a activar la reflexión sobre este tema junto a la sociedad. Ante la actual coyuntura de gobierno, con una mayor flexibilización en la autorización de ingredientes activos en el país, es necesario que el PARA sea fortalecido.

Agrotóxicos; Seguridad Alimentaria y Nutricional; Vigilancia Sanitaria
Recebido em 19/Jun/2019

Versão final reapresentada em 09/Jun/2020 Aprovado em 11/Jul/2020 